This report was prepared as an account of work sponsored by an agency of the United Stules Government. Neitber the United States Government oor any agency thereof, bor any of their employees, makes any warranty, express or impliod, or sssumes any legal liability or responsibility for the eccuracy, completeness, of usefulness of any informstion, apperatus, product, or process disclosed, or represcats that its use would not infringe privately owned rigbts. Reference herejn to aty apecific commercial product, process, or service by trade name, trademark, menufucturer, or aherwise does nor necesurity constitute or imply its endorsement, recommendation, or favoring by the United States Gowernaneal or any agency theteof. The views and opinions of authors expresed berein do sot necessarily state or reflect thoce of the United States Government or any stency thereof.

\title{
Pulse Length Assessment of Compact Ignition Tokamak Designs
}

\author{
D. P. Stotler and N. Pomphrey \\ Princeton Plasma Physics Laboratory \\ Princeton University \\ Princeton, New Jersey 08543
}

PPPL-2630

DE89 014601

\section{ABSTRACT}

A time-dependent zero-dimensional code has been developed to assess the pulse length and auxiliary heating requirements of Compact Ignition Tokamak (CIT) designs. By taking a global approach to the calculation, parametric studies can be easily performed. The accuracy of the procedure is tested by comparing with the Tokamak Simulation Code [S. C. Jardin, N. Pomphrey, and J. De Lucia, J. Comput. Phys., 66, 481 (1986)] which uses theory-based thermal diffusivities. A series of runs is carried out at various levels of energy confinement for each of three possible CIT configurations. It is found that for cases of interest, ignition or an energy multiplication factor $Q \gtrsim 7$ can be attained within the first half of the planned five-second flattop with $10-40 \mathrm{MW}$ of auxiliary heating. These results are supported by analytic calculations. 


\section{Introduction}

Present designs of the Compact Ignition Tokamak ${ }^{1}$ (CIT) are limited to a current flattop time of five seconds due to resistive heating of the toroidal field coils and other factors. Some of these five seconds will be needed to complete the plasma heating process begun during the current ramp phase. The rest of the flattop time will then be available for studying the physics of an ignited plasma. In this paper, we provide an estimate of the length of this period for three possible CIT configurations.

The time required to heat the plasma to ignition temperatures is directly related to the amount of auxiliary power available. So, we can equivalently view this study as yielding the level of auxiliary heating needed to obtain a certain value of the heating time.

Previous work along these lines employed complicated (and time-consuming) 1-1/2-D transport simulations. ${ }^{2.3}$ Since reliable transport models for this task do not exist in fully validated form, it is not clear that the extra effort required to proceed with these simulations would be worthwhile. Instead, we will employ a code designed to follow the time evolution of the volumeintegra ed plasma energy. This approach results in a significant decrease in the time needed to analyze a particular discharge scenario. Hence, a number of studies spanning the envisioned parameter space can be carried out easily.

As has been pointed out elsewhere (see Ref. 4 and references therein, for example), the level of energy confinement in CIT is the most uncertain element in this type of calculation. If the thermal insulation is below a certain amount, it will be difficult to attain values of the energy multiplication factor $Q$ much in excess of unity without extraordinary auxiliary heating power (say, > $40 \mathrm{MW}$ ). On the other hand, if the energy confinement is above some larger value, CIT should ignite readily with little or no auxiliary power input. The questions we wish to answer are relevant only in a band of energy confinement between these two extremes.

In this range, we find for each of the CIT designs that $Q \gtrsim 1$ or ignited operation can be achieved within the first half of the flattop for $10-40 \mathrm{MW}$ of auxiliary power. Detailed results are presented in a table.

Several aspects of this problem are amenable to analytic solution. We will present two simple models that show qualitatively how the time needed to 
heat the plasma depends upon the auxiliary power available, the minimum auxiliary power required, plasma density, etc. A formula for the minimum power required to achieve ignition has been developed by Waltz et al. ${ }^{5}$; we will apply it to our assumed energy confinement scaiing. Finally, we will consider the consequences of using an energy confinement formula that depends upon the input power rather than the plasma energy, as is assumed for the bulk of this work.

In Sec. II, we describe our global, time-dependent code and present a comparison with a sophisticated transport code. Section III contains the results of a parametric study for three different CIT designs. The analytic calculations are presented in Sec. IV. Finally, we provide a summary in Sec. V.

\section{Time-Dependent Global Code}

A number of global power balance codes have been developed recently. ${ }^{5-9}$ They typically solve an equation similar to

$$
P_{\alpha}+P_{O H}+P_{\text {aux }}=P_{\text {con }}+P_{\text {rad }} \text {. }
$$

The individual terms represent the volume-integrated contributions made to the total power balance by alpha, ohmic, and auxiliary heating; thermal conduction and radiated losses are on the right-hand side. We generalize this expression to include time dependence and helium ash accumulation:

$$
\begin{aligned}
& \frac{d W_{\text {tot }}}{d t}=-P_{\text {con }}-P_{\text {rad }}+P_{\alpha}+P_{O H}+P_{a u r}, \\
& \frac{d N_{H e}}{d t}=\frac{P_{\alpha}}{E_{\alpha}}-\frac{N_{H e}}{\tau_{p, H e}}
\end{aligned}
$$

The various terms in Eq. (2) will be described below. In Eq. (3), $N_{H e}$ is the number of helium ash particles, $E_{\alpha}=3.5 \mathrm{MeV}$ is the alpha birth energy, and $\tau_{p, H e}$ is the (constant) helium ash particle confinement time. Equation (3) assumes that the slowing down of fast alpha particles takes place instantaneously. In reality, the alpha slowing-down time in CIT is expected to be on the order of 100 msec. 
Codes such as the one described here are zero-dimensional $(0-D)$ in that the plasma profiles are all specified on input. We will use for density, temperature, and plasma current density

$$
x=x_{0}\left(1-r^{2} / a^{2}\right)^{a_{x}},
$$

where $x$ is replaced by $n, T$, and $J$, respec...., ; $a$ is the plasma minor radius. Then, the alpha power is computed using

$$
P_{\alpha}=E_{\alpha} 4 \pi^{2} R \kappa \int_{0}^{a} r d r n_{D} n_{T} \overline{\sigma v}_{D T}
$$

where $R$ is the plasma major radius, and $x$ is the plasma elongation. The reactivity, $\overline{\sigma v_{D T}}$, is calculated with a formula obtained by Hively ${ }^{10}$ in order to ensure correct results in all temperature regimes. Consequently, this integral must be computed numerically for each value of the density-weighted, volume-averaged temperature, $(T\rangle \equiv\left\langle n_{e} T\right\rangle /\left(n_{e}\right)$.

The ohmic heating power is (all powers are in watts)

$$
\begin{aligned}
& P_{\text {OH }}=\frac{4.17 \times 10^{3} Z_{\text {eff }} \ln \Lambda \gamma_{N C} T_{0}^{-3 / 2} V}{1+2 \alpha_{J}-\frac{3}{2} \alpha_{T}} \\
& \times\left\{\frac{B_{T}\left[1+\kappa^{2}\left(1+2 \delta^{2}-1.2 \delta^{3}\right)\right]}{2 \kappa R q_{0}}\right\}^{2},
\end{aligned}
$$

where $Z_{\text {eff }}$ is the effective charge, $\ln \Lambda$ is the Coulomb logarithm, ;NC is the neoclassical resistivity enhancement factor (constant, taken to be 2.5), $T_{0}$ is the central electron (and ion) temperature in $\mathrm{keV}, B_{T}$ is the toroidal magnetic field in tesla, $R$ and $a$ are in metres, $\delta$ is the triangularity, and $V$ is the plasma volume in $\mathrm{m}^{3}$. This expression for $P_{O H}$ is essentially the same as that used by Uckan. ${ }^{9}$

We will assume that the safety factor on axis, $q_{0}=1$ and compute $\alpha_{J}=$ $q_{c y l} / q_{0}-1$. For the equivalent cylindrical safety factor, we take

$$
q_{t y l}=\frac{5 a^{2} B_{T}}{R I_{p}} \frac{\left[1+\kappa^{2}\left(1+2 \delta^{2}-1.2 \delta^{3}\right)\right]}{2},
$$

whrre $I_{p}$ is the plasma current in mega-amperes. These units will be used taroughout this paper unless otherwise specified. 
Only bremsstrahlung radiation is included ${ }^{9}$ in $P_{\text {rad }}$ :

$$
P_{r a d}=5.31 \times 10^{-37} \frac{n_{e 0}^{2} T_{0}^{1 / 2} Z_{e f f} V}{\left(1+2 \alpha_{n}+\frac{1}{2} \alpha_{T}\right)},
$$

where $n_{\mathrm{eo}}$ is the central electron density.

Finally, the conducted losses are written as

$$
P_{\text {con }}=\frac{W_{\text {tot }}}{\tau_{E}}=\frac{2.40 \times 10^{-16}\left(\left\langle n_{\mathrm{e}} T\right\rangle+\left\langle n_{\mathrm{i}} T\right\rangle\right) \mathrm{V}}{\tau_{E}} .
$$

The time dependence of $P_{\text {aux }}$ (the auxiliary heating power), $Z_{e f f}, I_{p}, B_{T}$, $\left\langle n_{e}\right\rangle$, and the plasma boundary shape are specified on input. We assume that there is one impurity of charge $Z$ in addition to the helium ash. From the electron density, $Z_{\text {eff }}, Z$, and the helium ash density, we can compute the amount of hydrogen in the plasma, taken to be $50 \%$ deuterium and $50 \%$ tritium. Constant values of $\alpha_{n}$ and $\alpha_{T}$ are prescribed as well. Then, given initial conditions for $W_{\text {tot }}$ and $N_{\text {He }}$ Eqs. (2) and (3) can be integrated using standard techniques.

In order to estimate the accuracy of this procedure, we compare a simulation of CIT produced by the Tokamak Simulation Code ${ }^{11}$ (TSC) with results from our code. TSC is being used to evaluate the magnetics design for CIT. In addition to the free boundary equilibrium calculations required for this task, it carries out a complete time-dependent, 1-1/2-D transport calculation.

We need to make two minor modifications to our 0-D code to match features present in TSC. First, a global calculation of the cyclotron radiation is inclided in $P_{\text {rad. }}$. Second, a feedback loop is installed to adjust $P_{\text {aux }}$ to maintain a total input power $P_{\alpha}+P_{a u x}+P_{O H} \leq 110 \mathrm{MW}$. Neither of these are used in the calculations described in Sec. III.

The specific TSC simulation with which we will be comparing is designated as F11D1. It is similar to the one described in detail in Ref. 12. From the TSC output, we take the field and shape ramps, shown in Fig. 1. The auxiliary power programming is included in Fig. 2; $15 \mathrm{MW}$ of auxiliary power is input at $t=4.5 \mathrm{~s}$, and another $15 \mathrm{MW}$ is added at $t=7.5 \mathrm{~s}$. The falloff of $P_{\text {cur }}$ for $t>7.5 \mathrm{~s}$ is the result of the feedback loop explained above. The volume-averaged electron density is brought up linearly in time from $\left\langle n_{e}\right\rangle=0$ 
at $t=0$ to $\left\langle n_{e}\right\rangle=3.1 \times 10^{20} \mathrm{~m}^{-3}$ at $t=7.5 \mathrm{~s}$ and held constant thereafter, The density and temperature profiles TSC obtains do not vary greatly during the simulation. We choose our profile peaking factors using

$$
\frac{n_{0}}{\langle(n)}=1+\alpha_{n}
$$

and

$$
\frac{T_{0}}{\langle T\rangle}=\frac{\left(1+\alpha_{n}+\alpha_{T}\right)}{\left(1+\alpha_{n}\right)}
$$

arriving at $\alpha_{n}=0.58$ and $\alpha_{T}=1.53$. As in TSC, we employ $Z_{\text {eff }}=1.5$, $Z=8$, and $\tau_{p, H e}=1 \mathrm{~s}$.

It remains only to specily the form of $\tau_{E}$. TSC uses a generalization of the Tang transport model based on drift-wave turbulence. ${ }^{13.14}$ The TSC expression for the thermal diffusivity $\chi(r)$ consists of two pieces, one for the ohmic regime and one for the auxiliary heated regime. The two are combined in a sum of squares sense. To get a corresponding global model, we write

$$
\tau_{E}^{-2}=\tau_{N A}^{-2}+\left(c_{\tau} \tau_{\text {aux }}\right)^{-2},
$$

where $\tau_{a u x}$ is the scaling for auxiliary heated plasmas, and

$$
\tau_{N A}=7 \times 10^{-22} \bar{n}_{e} a R^{2} q_{c y l}
$$

is the neo-Alcator (ohmic) contribution, with $\bar{n}_{e}$ being the line-averaged electron density. The constant multiplier $c_{\tau}$ is specified on input.

We will use the Kaye-All-Complex L-mode scaling ${ }^{4,15}$ for $\tau_{a u r}$,

$$
\tau_{\text {aux }}=\tau_{E}^{K A C}=0.0521 \bar{A}_{i}^{0.5} \kappa^{0.25} I_{p}^{0.85} \bar{n}_{c, 19}^{0.1} B_{T}^{0.3} a^{0.3} R^{0.85} P_{i n}^{-0.5},
$$

where $\bar{A}_{i}$ is the average ion mass (taken to be 2.5 ): $\bar{n}_{e, 19}$ is the line-averaged electron density in units of $10^{19} \mathrm{~m}^{-3}$, and $P_{\text {in }}=P_{\alpha}+P_{O H}+P_{a u x}-P_{\text {rad }}$ is the net input power in megawatts. We intend for this $P_{\text {rad }}$ to refer only to centrally peaked radiation mechanisms, such as bremsstrahlung, that affect the power balance in the center of the plasma. We evaluate $\tau_{N_{A}}$ and $\tau_{E}^{K A C}$ at one point during the flattop of the TSC simulation and choose $c_{\tau}$ so that Eq. (10) yields the simulated value of $\tau_{E}$. This procedure leads to $c_{\tau}=1.9$.

The results of the comparison between the 0 -D model and TSC are shown in Fig. 2. We first note that the 0-D estimate of $P_{O H}$ is $\sim 1-2 \mathrm{MW}$ too 
large. Possible causes for this discrepancy are differences in current profile shape, flux surface shape, and neoclassical resistivity treatment. The total radiated power $P_{\text {rad }}$ matches very well throughout. The alpha power $P_{\alpha}$ is $\sim 10 \%$ larger in the 0-D code than in TSC. This is due to differences in the density and temperature profile shapes, as well as differences in the plasma volume. The conducted powers agree by virtue of the feedback loop on $P_{\text {aux }}$ and the choice of $c_{r}$.

The error in the power balance brought on by the difference in $P_{a}$ is absorbed by $P_{\text {avr }}$ via the feedback loop. In particular, note that $P_{\text {aux }}<0$ in the O-D code. From the point of view of Eq. (2), there is nothing peculiar about this. While physically unrealistic, it does allow us a clear comparison of the two simulations. In particular, we conclude that our error is about 10 MW out of $110 \mathrm{MW}$ total input power, $\sim 10 \%$.

\section{Parametric Study for CIT}

We examine three proposed magnetic configurations for CIT:

1. $I_{p}=9 \mathrm{MA}, B_{T}=8.2 \mathrm{~T}$ - present CIT design with existing TFTR power supplies.

2. $I_{\mathrm{p}}=11 \mathrm{MA}, B_{\mathrm{T}}=10 \mathrm{~T}-$ present CIT design with upgraded power supplies.

3. $I_{p}=13 \mathrm{MA}, B_{T}=11.8 \mathrm{~T}$ - possible enhanced design using a bucked coil design.

For all three designs, $R=2.1 \mathrm{~m}, a=0.65 \mathrm{~m}, \kappa=2$, and $\delta=0.4$. The magnetic fields have been chosen in cases (1) and (3) to yield the same value of $q_{c y l}$ as in case (2), $q_{\text {eyl }}=2.73$. We take the field and shape ramps for the 11 MA device from the TSC run F11D1 (Fig. 1) and rescale the $I_{p}$ and $B_{T}$ programming for the other two cases.

The input parameters are as in Sec. II with the following exceptions. The energy confinement time is written as

$$
\tau_{E}=\min \left[\tau_{\mathrm{N}_{A}}, c_{r}^{2} \tau_{E}^{K A C}\left(W_{t o t}\right)\right] .
$$


This expression is more appropriate since the database from which $T_{E}^{h A C}$ is derived contains numerous low power discharges. ${ }^{16}$ The neo-Alcator contribution is included only to provide a reasonable behavior when $\left\langle n_{e}\right\rangle$ and $(T) \rightarrow 0$. We have also rewritten the scaling in torms of the plasma energy instead of the input power. The means of doing this and its consequences will be described in Sec. IV.C. The multiplier $c_{\tau}$ is squared in Eq. (13) as a result of the conversion procedure. It is still a linear multiplier on the power form of $\tau_{E}^{K A C}$. In the results described below, $c_{r}$ will be varied systematically.

Another difference with Sec. II is that instead of assuming a step function increase, we ramp the auxiljary beating power linearly with time between $t=4.5 \mathrm{~s}$ and $5.5 \mathrm{~s}$. This is a more plausible representation of the increase in heating efficiency expected to take place as the ICRF resonance.layer moves toward the plasma center during the magnetic field ramp. The auxiliary power is held constant thereafter. We can vary the amount of heating and the length of time it is on. For ignited cases, we remove the auxiliary heating once the discharge has reached the point of ignition. For slightly sub-ignited cases, we suddenly reduce $P_{\text {aux }}$ at some point during the current flattop in order to operate at $Q \gg 1$. In all other instances, the auxiliary heating remains at full power until the end of the run.

As will be demonstrated in more detail in Sec. IV.B, the performance of CIT is very sensitive to the value of $c_{r}$. Given that it would be unrealistic to plan for much more than $40 \mathrm{MW}$ of auxiliary power on CIT, we are restricted in how small $c_{T}$ can be in order to attain $Q>5$ operation. Below this level of ionfinement, the most desirable operating point (with $P_{\text {aux }} \leq 40$ $M W$ ) is at a sufficiently low temperature that the time needed to heat the plasma is short. Likewise, above some larger value of $c_{r}$, ohmic ignition is possible. In this case, the time required to reach ignition, perhaps with a small amount of auxiliary power, is short enough that it is, again, pointless to do the calculation. We examine three values of $c_{r}$ lying between these two extremes.

For each $c_{r}$, we consider separately two values of $P_{\text {aux }}, 10 \leq P_{\text {aux }} \leq 40$ $M W$, in order to give some idea of the range of modes of operation possible at that level of confinement. We pick the flattop $\left\langle n_{e}\right\rangle$ to yield the highest $Q$ or quickest ignition at each auxiliary power level. We require that the 
line-averaged density be less than ${ }^{17}$

$$
\bar{n}_{e, \max }=\frac{2 B_{T}}{R q_{e}} 10^{20} \mathrm{~m}^{-3},
$$

where $q_{e}=5 a^{2} \kappa B_{T} / R I_{\mathrm{p}}$ is the engineering $q$ value. As in the TSC comparison, the density is ramped linearly from zero between $t=0$ and $t=7.5$ s.

For each of the three CIT configurations, there are three $c_{\tau}$ values, each run with two levels of auxiliary heating. This makes a total of 18 simulations. The results are summarized in Table I. The first three columns provide the Lmode multiplier $c_{r}, P_{u u=}$ in $\mathrm{MW}$, and the flat top density in units of $10^{20} \mathrm{~m}^{-3}$, $\left\langle n_{e, 20}\right\rangle$. The maximum values of $P_{\alpha}$ and $Q$ occurring during the simulations are given. Ignited cases are indicated by $Q_{\max }=\infty$; the time at which the auxiliary heating is turned off is also given for these runs. The time of maximum $P_{\alpha}$ (ignited cases) is presented in the last column. As will be demonstrated in Sec. IV.A, a finite $Q$ state is approached logarithmically in time (ignoring He ash buildup), and much of the flattop is spent attaining the last few increments in $Q$. So, we take the $90 \% Q_{\max }$ point as characterizing the time required to heat the plasma in these cases. The $Q \gg 1$ simulations are distinguished by the additional entry "@ $x \mathrm{MW}$ in the $Q_{\max }$ column. For these runs, $P_{\text {aux }}$ is reduced to $x \mathrm{MW}$ at some point prior to $t\left(0.9 Q_{\max }\right)$ and held there for the remainder of the discharge.

As examples, we present in Figs. 3 - 5 results for the 11 MA configuration with $c_{r}=1.6$. In Fig. 3 , the contours of constant auxiliary power (in MW) required to maintain steady state at a given $\left\langle n_{e}\right\rangle$ and $\langle T\rangle$ are plotted (see, for example, Refs. 6 and 9). This type of diagram is referred to as a Plasma OPeration CONtour or POPCON plot. ${ }^{18}$ The density and $\beta$ limits $\left(\beta_{\max }(\%)=3 I_{p} / a B_{2}\right)$ are also indicated. Figure 3 is constructed using the flattop parameters (i.e., $t \geq 7.5 \mathrm{~s}$ ) and does not assume any helium ash buildup. For this $c_{r}$, we examine $P_{a u x}=20$ and $30 \mathrm{MW}$. The densities for these cases are $\left\langle n_{e, 20}\right\rangle=3.8$ and 4.3 , respectively.

The time dependence of the terms in Eq. (2) for the $20 \mathrm{MW}$ case is shown in Fig. 4. According to Fig. 3, this case should be ignited. But, there is sufficient helium ash buildup $\left(n_{\alpha} / n_{e}=0.014\right.$ at $\left.t=12 \mathrm{~s}\right)$ during the discharge to close the relatively small ignition window present at this density. However, $Q \gg 1$ operation is possible. So, when $\langle T\rangle$ reaches $\sim 9$ 
$\mathrm{keV}(t=8.6 \mathrm{~s})$, we reduce $P_{\mathrm{aux}}$ to $2 \mathrm{MW}$. At the end of the run, $\langle T\rangle=9.7$ $\mathrm{keV}$.

By raising the density to the maximum allowed, $\left\langle n_{e}\right\rangle=4.3 \times 10^{20} \mathrm{~m}^{-3}$, we can achieve ignition at this value of $c_{T}$ (Fig. 5). To overcome the increased thermal inertia without having to heat through most of the flattop, however, we need to raise $P_{\text {aurs }}$ to $30 \mathrm{MW}$. We turn off the auxiliary heating when $\langle T\rangle \simeq 9 \mathrm{keV}$. Again, the helium ash buildup ends the ignition soon after it starts. By the end of the calculation, $(T)$ drops to $8.5 \mathrm{keV}$.

With the freedom to vary $P_{\text {aux }}$ between 10 and $40 \mathrm{MW}$ and to choose any operating density below the prescribed density limit, we have been able to reach ignition (for the larger $c_{\tau}$ cases) or at least $Q \gtrsim 7$ within the first half of the flattop $(t<10 \mathrm{~s})$ in most of the cases reported in Table I. Greater restrictions on the auxiliary power available, the density, the magnitude of $c_{7}$, or on the fusion power could lead to scenarios in which the full flattop time is needed to reach the desired operating point.

\section{Analytic Calculations}

Several aspects of this problem can be addressed analytically. We will develop two simple models to describe the time required to heat the plasma in terms of quantities found on a POPCON plot. By applying the results of Waltz, Dominguez, and Perkins ${ }^{5}$ to the particular cases of interest here, we can understand why we find CIT performance to be so sensitive to $c_{4}$. This same result can be used to show that if ignition can be achieved at all in CIT for these confinement and density scalings, the power required to reach ignition is $\lesssim 10 \mathrm{MW}$. Finally, we will demonstrate that the use of a power-dependent confinement scaling instead of one written in terms of the plasma energy can almost double the required heating time.

\section{IV.A. Time Required to Heat the Plasma}

It is difficult to integrate Eq. (2) analytically in general. Some of the problem terms can be neglected, but the results still tend to be too complicated to provide insight. What we seek to do here instead is to model the most important structure displayed in POPCON plots (e.g., Fig. 3) in 
such a way that Eq. (2) can be integrated exactly, yielding $W_{\text {tot }}(t)$ or at least $t\left(W_{\text {tot }}\right)$

We first consider cases in which ignition is possible within the prescribed density and $\beta$ limits, Fig. 3 for example. The precise question we seek to answer is: how long does it take to heat from the ohmic equilibrium contour to the ignited equilibrium contour? This is, of course, dependent upon the path in $\left\langle n_{e}\right\rangle$ and $\langle T\rangle$ space.

We wiil assume for the moment that all parameters except for $\left\langle n_{e}\right\rangle$ and $\langle T\rangle$ are fixed during this process. Then, all of the variations in $d W_{t o t} / d t$ can be deduced from a PCPCON diagram, i.e., a contour plot of

$$
P_{p b}\left(\left\langle n_{e}\right\rangle,\langle T\rangle\right) \equiv \frac{W_{\text {tot }}}{\tau_{E}}+P_{\text {rad }}-P_{\alpha}-P_{O H} .
$$

The subscript "pb" refers to power balance. In an ignited case, $P_{p b}$ goes through a maximum along a constant denzity path between the ohmic and igaited equilibrium contours. We can model this behavior at least near the maximum with a parabola,

$$
P_{p b}=a\langle T)^{2}+b(T\rangle+c . \quad .
$$

By specifying $P_{p b}$ at iwo points (for example, one on each of the ohmic and ignited equilibrium contours) and the maximum value, $P_{m}$, of $P_{p b}$ along the path, the coefficients $a, b$, and $c$ can be determined.

In order to generalize this procedure slightly, we write instead

$$
P_{p b}=a W_{t o t}^{2}+b W_{t o t}+c .
$$

This allows for cases in which the density or even some of the other parameters in the calculation are varying in time, during the current ramp phase of CIT for example. If most of the heating is done during the flattop, we can still use a POPCON to obtain an estimate of $P_{m}$.

We now assume we know $P_{p b}\left(W_{0}\right)=P_{0}$ and $P_{p b}\left(W_{1}>W_{0}\right)=P_{1}$ and the maximum value of $P_{p b}$ between $W_{0}$ and $W_{1}, P_{m}$. We allow $P_{0}$ and $P_{1}$ to be nonzero to account for cases in which ohmic ignition is possible. If the system has energy $W_{i}$ at time $t_{i}$, and a constant auxiliary power $P_{\text {aux }}$ is applied until the energy reaches $W_{f}$ at time $t_{f}$, we can integrate Eq. (2) 
using Eqs. (15) and (17) to obtain

$$
\begin{aligned}
t_{f}-t_{i}=\frac{W_{1}-W_{a}}{\sqrt{\Delta P_{a}}\left(\sqrt{\Delta P_{0}}+\sqrt{\Delta P_{1}}\right)} \\
\times\left[\operatorname { t a n } ^ { - 1 } \left\{\left(\sqrt{\frac{\Delta P_{a}}{\Delta P_{a}}}+\sqrt{\frac{\Delta P_{1}}{\Delta P_{a}}}\right)\left[\frac{W-W}{W_{1}-W_{0}}\right]\right.\right. \\
\left.\left.\quad+\frac{1}{2}\left(\sqrt{\frac{\Delta P_{1}}{\Delta P_{a}}}-\sqrt{\frac{\Delta P_{0}}{\Delta P_{a}}}\right)\right\}\right]_{W}^{W_{i}},
\end{aligned}
$$

where

$$
\begin{aligned}
W & \equiv\left(W_{0}+W_{1}\right) / 2 \\
\Delta P_{0} & \equiv P_{\mathrm{m}}-P_{0} \\
\Delta P_{1} & \equiv P_{\mathrm{m}}-P_{1} \\
\Delta P_{\mathrm{a}} & \equiv P_{\mathrm{aux}}-P_{\mathrm{m}} .
\end{aligned}
$$

In the limit of $P_{a u x} \gg P_{m}$, Eq. (18) becomes

$$
t_{f}-t_{i} \simeq \frac{W_{f}-W_{i}}{P_{\text {cux }}}
$$

as expected.

If we take Eq. (17) to hold for all $W_{\text {tot, }}$ as if $P_{\alpha}$ would continue to dominate $P_{\text {con }}$ with increasing $T, W_{\text {tot }} \rightarrow \infty$ in a finite time. This defines a characteristic time to heat to ignition,

$$
t_{f}-t_{i} \sim \frac{\pi}{2} \frac{W_{1}-W_{0}}{\sqrt{\Delta P_{a}}\left(\sqrt{\Delta P_{0}}+\sqrt{\Delta P_{1}}\right)} .
$$

In this expression, $\Delta P_{a}$ represents the excess input power; $\Delta P_{0}$ and $\Delta P_{1}$ describe the steersess of $P_{p b}\left(W_{t o t}\right)$ and are thus directly related to the rate at which the thermal instability proceeds.

Typical numbers for an ignited CIT would be (as in Fig. 3) $W_{f}-W_{i} \sim$ $W_{1}-W_{0} \sim 50 \mathrm{MJ}, P_{1} \simeq P_{0} \simeq 0$, and $P_{m} \simeq 10 \mathrm{MW}$. For convenience we have set $W_{i}=W_{0}$ and $W_{f}=W_{1}$. With these values and $P_{a u x}=20 \mathrm{MW}$, Eq. (18) and Eq. (20) yield exactly the same result, $t_{f}-t_{i}=4 \mathrm{~s}$. 
For most ignited CIT cases, $P_{\mathrm{m}}<10 \mathrm{MW}$ (see Sec. IV.B), and we may even have $P_{m} \lesssim 0$ (ohrnic ignition). In this case, Eq. (19) provides an approximate lower limit to the time required to heat the plasma: $t_{f}-t_{i} \simeq 2.5$ s.

Returning now to Table $I$, using $t_{i} \simeq 5 \mathrm{~s}$, and setting $t_{f}$ in the ignited cases equal to the heating off time, we see that the range $t_{j}-t_{i}=2.5-4 \mathrm{~s}$ also describes our numerical calculations fairly well.

We now consider cases with finite $Q$. In this instance, the path from ohmic equilibrium to the final state is one of monotonically increasing $P_{p b}$, as in the moderate-density, high-temperature region of Fig. 3. For small $c_{\tau}$, the conducted losses dominate for all temperatures above ohmic equilibrium provided $\left\langle n_{e}\right\rangle$ is not so large as to make radiation dominant. In the limit of $c_{T} \rightarrow 0, P_{p b} \propto\langle T\rangle^{2}$ (assuming $\tau_{E} \propto P_{i n}^{-0.5}$ ). The finite $Q$ cases examined in Table I have more moderate $c_{r}$. For then, the alpha power effectively reduces the rate of rise of $P_{p b}$ below $(T)^{2}$.

We model these cases qualitatively by linea: $y$ interpolating between two points on the POPCON. We specify the plasma energy at ohmic equilibrium, $W_{\text {OH, }}$, and the energy attained in thr limit $t \rightarrow \infty$ for a given $P_{\text {aux }}, W_{P_{\text {aux }}}$. This,

$$
P_{p b}\left(W_{\text {tot }}\right)=\left(\frac{W_{\text {tot }}-W_{O H}}{W_{P_{\text {aux }}}-W_{O H}}\right) P_{\text {aux }} .
$$

Integration of Eq. (2) using Eqs. (15) and (21) with initial and final energies $W_{i}$ and $W_{f}$, respectively, yields

$$
t_{f}-t_{i}=\frac{W_{P_{\text {axx }}}-W_{O H}}{P_{\text {aux }}} \ln \left(\frac{1-W_{i} / W_{P_{\text {aus }}}}{1-W_{f} / W_{P_{\text {aux }}}}\right) .
$$

Note that the time required to heat the plasma diverges logarithmically as $W_{f}$ approaches $W_{P_{\text {oux }}}$.

To provide some typical values for these quantities, we give in Table II data from the $Q \sim 7-10$ runs of Sec. III. We evaluate $t_{i}$ and $W_{O H}$ at the earliest time for which $\dot{P}_{\text {aux }}>P_{O H}$. Since $Q$ increases roughly like $W_{\text {tot }}^{2}$, we assume $W_{f}=0.95 W_{P_{a u s}}$ in Table II so that we can compare with the $t\left(0.9 Q_{\text {max }}\right.$, entry in Table I. This is meant only as a qualitative somparison since the above model is considerably simpler th $2 n$ the one used in the actual numerical calculations. 
In Table II, we see that, the "time required to heat the plasma" is roughly 2.5 to $3.5 \mathrm{~s}$, as was the case for the ignited discharges. We reiterate that this is the result of judicious choices of $\left\langle n_{e}\right\rangle$ and $P_{\text {aur }}$ and does not necessarily indicate any fundamental limitation. The effects of these choices are evident in each of Eqs. (18) $-(20)$, and (22). The thermal inertia of the plasma increases linearly with $\left(n_{e}\right)$, and the heating time decreases uniformly with $P_{\text {auz. }}$ Between these two parameters, there is enough freedom to arrange for $t_{f}-t_{i}$ to fall within the range desired for CIT.

\section{IV.B. Saddle Point Conditions}

POPCON diagrams computed for CIT with the Kaye-All-Complex scaling typically yield $P_{m}<10 \mathrm{MW}$ when the minimum density for ignition is below the density limit. We now use $P_{m}$ to refer specifically to the power required to maintain steady state at the saddle point $t^{6,9,18}$ of the POPCON. This is the absolute minimum power required for ignition. By applying the results of Waltz, Dominguez, and Perkins ${ }^{5}$ to the present situation, we can demonstraie that this relatively small value of $P_{m}$ is a general result.

In the calculation described in Ref. 5, $P_{O H}$ is neglected, a simplified expression for $P_{\alpha}$ is used (we insert the formula proposed in Ref. 9), and the energy confinement time is taken to be $\tau_{E} \propto\left\langle n_{e}\right)^{\zeta}\langle T\rangle^{-\xi}$. The system of equations $P_{m}=P_{p b}, \partial P_{p b} / \partial\left\langle n_{e}\right\rangle=0, \partial P_{p b} / \partial\langle T\rangle=0$ is solved analytically for the temperature, density $\left\{n_{e}\right\rangle_{m}$, and auxiliary power $P_{m}$ at the saddle point.

For Kaye-All-Complex scaling, $\zeta=-0.8$ and $\xi=1$ (see Sec. IV.C). Then,

$$
\left\langle n_{e}\right\rangle_{m}=206 .\left[c_{\tau}^{2}\left(\frac{B_{T}}{10 \mathrm{~T}}\right)^{0.6}\left(\frac{I_{p}}{11 \mathrm{MA}}\right)^{1.7}\right]^{-5} 10^{20} \mathrm{~m}^{-3}
$$

and

$$
P_{m}=3.06 \times 10^{4}\left[c_{\tau}^{2}\left(\frac{B_{T}}{10 \mathrm{~T}}\right)^{0.6}\left(\frac{I_{p}}{11 \mathrm{MA}}\right)^{1.7}\right]^{-10} \mathrm{MW}
$$

The coefficients in front of each of these expressions is a complicated function of $\alpha_{n}$ and $\alpha_{T}$; we have evaluated them at $\alpha_{n}=0.58$ and $\alpha_{T}=1.53$, the values used in Sections II and III. However, all of the $I_{p}, B_{T}$, and $c_{\tau}$ dependence appears explicitly in Eqs. (23) and (24). 
If $c_{T}$ (or $I_{p}$ and $B_{T}$ ) is too small, $\left\langle n_{e}\right\rangle_{m}$ is greater than the density limit value $\left\langle n_{e}\right\rangle_{\text {max }}$, and there is no window for ignited operation. Combining Eqs. (23) and (24) with this requirement, we obtain

$$
P_{m}<0.72\left(\frac{\left\{n_{e}\right\}_{\max }}{10^{20} \mathrm{~m}^{-3}}\right)^{2} .
$$

For CIT, the density limit scaling given in Eq. (14) typically yields $\left\langle n_{e}\right\rangle_{\max } \sim$ $2.5 \times 10^{20} \mathrm{~m}^{-3}$, depending on $I_{\mathrm{p}}$ and the density profile shape. Then, $P_{\mathrm{m}} \sim 3$ - $18 \mathrm{MW}$. However, this analysis has neglected $P_{O H}$. Near the saddle point, $P_{O H}$ is on the order of $5 \mathrm{MW}$ for CIT. As a lowest order correction, we subtract this from $P_{m}$ to estimate the auxiliary power required in steady state at the saddle point: $\sim-2-13 \mathrm{MW}$, in agreement with typical POPCON diagrams.

Another piece of information we can get out of Eqs. (23) and (24) is the approximate scaling of critical $c_{\zeta}$ values with $I_{p}$ :

$$
c_{\tau} \propto I_{p}^{-0.85} B_{T}^{-0.3} \text {. }
$$

As an example, assume that $c_{T, 11}$ is required to attain some particular level of performance for the $11 \mathrm{MA}$ CIT design. Then, a multiplier of $1.3 c_{r, 11}$ is needed to obtain roughly the same performance from the 9 MA configuration. Likewise, $0.8 c_{r, 11}$ becomes the critical value for the $13 \mathrm{MA}$ machine. This trend is evident in the stagger of the $c_{\tau}$ values between the various machines in Table I. Note that Eq. (26) is nothing more than the requirement that $T_{E}$ remain constant when $I_{p}$ and $B_{T}$ are changed.

Equations (23) and (24) exhibit a high sensitivity to $c_{7}$, and to a lesses extent, $I_{p}$ and $B_{T}$. The exponents " 5 " and " $10^{\prime}$ " in these equations correspond to $1 /(1+\zeta)$ and $2 /(1+\zeta)$ in the original formulas. We can see how this arises by writing $\zeta=-1+\epsilon$. Hence (see Sec. IV.C),

$$
\frac{P_{\text {con }}}{P_{\alpha}-P_{\text {rad }}} \propto \frac{\left\langle n_{e}\right)^{-\epsilon}}{c_{T}^{1+\xi}} .
$$

When $\epsilon \ll 1$, as is the case here, a small change in $c_{\tau}$ can be offset only by a large change in $\left\langle n_{e}\right\rangle$. Namely, to keep the ratio in Eq. (27) constant, we would require

$$
\left(n_{e}\right) \propto\left(c_{\tau}^{i+\xi}\right)^{\frac{-1}{1+\zeta}}
$$


as in Eq. (23).

Consequently, the density scaling of $\tau_{E}$ is critical. The exponent $\zeta$ changes greatly in a relative sense between the various L-mode scalings (see Refs. 9 and 15, for example). The values of $\zeta$ used in the Waltz, Doninguez, and Perkins drift wave-based models are completely different: $\zeta=0$ (ion temperature gradient driven instability) and $\zeta=1$ (dissipative trapped electron mode). Hence, we must be careful in interpreting the resuits of this paper. In particular, the detailed values of $c_{\tau}$ employed are highly dependent upon our use of the Kaye-All-Complex formula. Rather than viewing the 2.5 $4 \mathrm{~s}$ heating time calculated in Secs. III and IV.A as pertaining to only our particular confinement scaling, we should look at it as characteristic of all scenarios having similar values of $W_{f}-W_{i}$ and $P_{m}$, regardless of the $\tau_{\varepsilon}$ expression used.

\section{IV.C. Energy vs. Power Form of $\tau_{E}$}

Theories of tokamak energy confinemeric generally express their results in terms of local plasma parameters, $n_{\varepsilon}$ and $T$. It is for this reason that we have been using the energy form of $\tau_{E}$ up to this point. On the other hand, experimental values of $\tau_{E}$ are most readily categorized by input power, $P_{i n}$, and line-averaged density since they can be obtained with relatively little data analysis. In steady state, the two forms of $\tau_{E}$ are related by

$$
P_{\text {in }}=\frac{W_{\text {tot }}}{\tau_{E}}
$$

This is equivalent to Eq. (1) when we interpret $P_{\text {in }}$ as the net input power: $P_{\text {in }}=P_{\text {oH }}+P_{\alpha}+P_{\text {aur }}-P_{\text {rad }}$.

With

$$
\tau_{E}\left(P_{\text {in }}\right)=f_{+} P_{\text {in }}^{-\gamma},
$$

where $f_{r}$ contains all of the nonpower dependence (including the multiplier $\left.c_{-}\right)$, this procedure yields

$$
\tau_{E}\left(W_{\text {eot }}\right)=\left(f_{r} W_{\text {tot }}^{-\gamma}\right)^{1 /(1-\gamma)} .
$$

By definition a steady-state calculation of the power balance such as a POP. CON is indifferent as to which of these two forms is used. This is not the case when transient effects are of interest. 
We now define $\dot{W}_{\text {tot }}$ as the value of $d W_{\text {tot }} / d t$ obtained with $\tau_{E}\left(P_{\text {in }}\right)$, and $\dot{W}_{\text {tot }}^{\prime}$ as that found with $\tau_{E}\left(W_{t o t}\right)$. We can then show

$$
\frac{\tau_{E}\left(W_{t o t}\right)}{W_{t o t}} \dot{W}_{t o t}=1+\frac{\tau_{E}\left(W_{t o t}\right)}{W_{t o t}} \dot{W}_{t o t}^{\prime}-\left[1+\frac{\tau_{E}\left(W_{t o t}\right)}{W_{t o t}} \dot{W}_{t o t}^{\prime}\right]^{\gamma}
$$

In the limit $\frac{r g\left(W_{\text {tor }}\right)}{W_{\text {tod }}} \dot{W}_{\text {tot }}^{\prime} \ll 1$ (the time rate of change of the plasma energy is much less than the conducted losses), we can expand the term in brackets to obtain

$$
\dot{W}_{\text {tot }} \simeq \dot{W}_{\text {tot }}^{\prime}(1-\gamma) \text {. }
$$

In the opposite limit (valid when $P_{\text {aur }} \gg P_{\text {con }}$ ), $\dot{W}_{\text {tot }} \simeq \dot{W}_{\text {tot }}^{\prime}$.

For the cases considered in Sec. III, the first limit is more appropriate. Noting that $\gamma=0.5$ for the Kaye-All-Complex scaling, we see that $W_{t o t}$ would be reduced by a factor of two if we were to use the power form of $\tau_{E}$. In other words, it would take twice as long to increase $W_{\text {tot }}$ by a specific amount.

We show in Figs. 6 and 7 calculations identical to those given in Figs. 4 and 5 , respectively, but with the power form of $\tau_{E}$ replacing the energy form. The "heating off times" have been altered to yield the same maximum $P_{\alpha}$. Note that the total heating times required with the power form are less than double that needed with the energy form due to finite values of $\frac{T_{g}\left(W_{\text {tot }}\right)}{W_{\text {tou }}} \dot{W}_{\text {tot }}^{\prime}$.

In Sec. III, we were able to arrange the parameters of the various simulations so that the desired plasma operation point could be reached within the first half of the flattop. The above result then suggests that with the power form of $\tau_{E}$, we could still achieve roughly the same end states under the assumptions of Sec. III by the end of the flattop period. By examining experimental data, it may be possible to demonstrate that one of these two ways of writing $\tau_{E}$ is more appropriate than the other.

\section{Summary}

In summary, we have made an assessment of the pulse length and auxiliary heating requirements for three possible CIT designs. To this end, a timedependent 0-D. code has been developed. Our procedures have been checked by comparing against a TSC simulation of CIT. In this comparison, we have 
found that the flattop power balance matches to within $\div 10 \mathrm{MW}$ out of $\sim 100 \mathrm{MW}$ for each of the dominant terms.

The primary results of this paper are contained in Table I where we have presented parameters from several simulations of each of the suggested CIT magnetic configurations. The most critical quantity in determining CIT performance is the L-mode multiplier, $c_{+}$, defined in Eq. (13).

We can attempt to summarize Table $I$ as follows. For the smallest values of $c_{r}$ examined in each machine, $P_{\text {aur }}=40 \mathrm{MW}$ is required to achieve $P_{\sigma} \sim 60$ MW. While $P_{\mathrm{aur}}=20 \mathrm{MW}$ is sufficient to reach similar values of $Q, Q \gtrsim 7$, the resulting $P_{\alpha}$ is, of course, proportionately smaller. For the larger values of $c, Q \gg 1$ or ignited operation is obtained with $P_{a u x} \leq 20 \mathrm{MW}$. The freedom to adjust $P_{\text {aux }}$ and the plasma density has allowed us to reach these final plasma operating states during the first half of the current flattop.

Several analytic calculations have been presented. We first described simple models for the time required to heat the plasma, one for ignited cases and one for finite $Q$ operation. In both instances, application of the formulas to typical CIT scenarios yielded heating times of $2.5-4 \mathrm{~s}$, in qualitative agreement with Table I.

We then examined the minimum power required to reach ignition using expressions derived by Waltz, Dominguez, and Perkins. ${ }^{5}$ For the Kaye-AllComplex $\tau_{E}$ scaling, we were able to show that this auxiliary heating power is $P_{\text {aur }} \lesssim 10 \mathrm{MW}$. The equations used to do this demonstrate a high sensitivity to the value of $c_{r}$.

This sensitivity is directly related to the density scaling exponent of $\tau_{E}$. Since this quantity is somewhat uncertain, ${ }^{15}$ we conclude that the detailed results of Table I should be used with caution. Our numerical results are, however, applicable in a qualitative sense to other situations providing a similar level of energy confinement.

Finally, we have considered the consequences of writing $\tau_{E}$ in terms of the input power rather than the plasma energy, as was done throughout the rest of the paper. We have found that with the former, the time required to heat the plasma through a given range of plasma energy is almost twice as long as for the latter. Since the simulations of Sec. III required less than about $4 \mathrm{~s}$ to heat to the desired operating point, we would still be able to reach roughly the same final states by the end of the flattop even if this more 
pessimistic scaling is the appropriate description of the plasma.

\section{Acknowledgments}

The authors would like to acknowledge the numerous comments on this work provided by the CIT team. This work was supported by U.S. DOE Contract No. DE-AC02-76-CHO-3073. 


\section{References}

${ }^{1}$ G. BATEMAN et al., "CIT Physics and Engineering Basis," Proc. 12th Int. Conf. Plasma Physics and Controlled Nuclear Fusion, Nice, France, November 1988, paper IAEA-CN-50/G-2-1, International Atomic Energy Agency; see also J. A. SCHMIDT and H. P. FURTH, "Compact Ignition Tokamak Conceptual Design Report," A-860606-P-01, Princeton Plasma. Physics Laboratory (June 1986).

${ }^{2}$ D. P. STOTLER and G. BATEMAN, "Time-Dependent Simulations of a Compact Ignition Tokamak," Fusion Technol. 15, 12 (1989).

${ }^{3}$ C. E. SINGER, L.-P. KU, and G. BATEMAN, "Plasma Transport in a Compact Ignition Tokamak," Fusion Technol. 13, 543 (1988).

'D. P. STOTLER, R. J. GOLDSTON, and the CIT Team, "Ignition Probabilities for CIT," Princeton Plasma Physics Laboratory Report No. PPPL-2629 (1989); Fusion Technol. (to be published).

${ }^{5}$ R. E. WALTZ, R. R. DOMINGUEZ, and F. W. PERKINS, "Drift Wave Model Tokamak Ignition Projections with a Zero-Dimensional Transport Code," Nucl. Fusion 29, 351 (1989).

${ }^{B} \mathrm{~N}$. A. UCKAN and J. SHEFFIELD, "A Simple Procedure for Establishing Ignition Conditions in Tokamaks," Tokamak Startup, p. 45, H. KNOEPFEL, Ed., Plenum Press, New York (1986).

'Y. C. SUN, D. E. POST, G. BATEMAN, and D. STOTLER, "The Performance of CIT and ITER Under Various Scaling Laws," Bull. Am. Phys. Soc. 33, 1971 (1988).

${ }^{8}$ O. MITARAI, A. HIROSE, and H. M. SKARSGARD, "Generalized Ignition Contour Map and Scaling Law Requirement for Reaching Ignition in a Tokamak Reactor," Nucl. Fusion 28, 2141 (1988).

${ }^{9}$ N. A. UCKAN, "Relative Merits of Size, Field, and Current on Ignited Tokamak Performance," Fusion Technol. 14, 299 (1988).

${ }^{10}$ L. M. HIVELY, "Convenient Computational Forms for Maxwellian Reactivities," Nucl. Fusion 17, 873 (1977). 
113. C. JARDIN, N. POMPHREY, and J. DE LUCIA, J. Comput. Phys 66, 481 (1986).

${ }^{12} N$. POMPHREY, "The 11 MA Fiducial Discharge," Compact Ignition Tokamak Report AE-890417-PPL-04 (1989).

${ }^{13}$ S. C. JARDIN, J. DE LUCIA, M. OKABAYASHI, N. POMPHREY, M. REUSCH, S. KAYE, and H. TAKAHASHI, "Post-Disruptive Plasma Loss in the Princeton Beta Experiment (PBX)," Nucl. Fusion 27, 569 (1987).

1"W. M. TANG, "Microinstability-Based Model for Anomalous Thermal Confinement in Tokamaks," Nucl. Fusion 26, 1605 (1986).

${ }^{15}$ S. M. KAYE, "Survey of Energy Confinement Scaling Expressions," presented at the ITER Specialists' Meeting on Enemy Confinement, 24-27 May 1988, Garching, Federal Republic of Germany.

${ }^{16}$ S. M. KAYE, Princeton Plasma Physics Laboratory, Private Communication (1989).

${ }^{17}$ JET Team, "JET Latest Results and Future Prospects," Proc. 12th Int. Conf. Plesma Physics and Controlled Nuclear Fusion, Nice, France, November 1988, paper IAEA-CN-50/A-1-3, International Atomic Energy Ageacy.

${ }^{18}$ W. A. HOULBERG, S. E. ATTENBERGER, and L. M. HIVELY, "Contour Analysis of Fusion Reactor Plasma Performance," Nucl. Fusion 22, 935 (1982). 


\begin{tabular}{|c|c|c|c|c|c|c|}
\hline & $P_{\text {aur }}$ & $\left\langle n_{e, 20}\right\rangle$ & $P_{a, \max }$ & $Q_{\max }$ & $\begin{array}{l}\text { heating } \\
\text { off time }\end{array}$ & $\begin{array}{c}t\left(P_{a, \max }\right) \text { or } \\
t\left(0.9 Q_{\max }\right)\end{array}$ \\
\hline \multicolumn{7}{|c|}{$I_{p}=9 \mathrm{MA}$} \\
\hline \multirow[t]{2}{*}{$c_{r}=1.6$} & 20 & 2.2 & 31 & 7.7 & - & 8.7 \\
\hline & 40 & 3.2 & 69 & 8.6 & + & 8.3 \\
\hline \multirow[t]{2}{*}{$c_{r}=1.9$} & 10 & 2.6 & 44 & 22. & 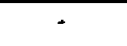 & 11.3 \\
\hline & 20 & 3.5 & 82 & 41. (@ $10 \mathrm{MW}$ ) & - & 9.6 \\
\hline \multirow[t]{2}{*}{$c_{\tau}=2.2$} & 10 & $\overline{3.5}$ & 56 & $\infty$ & 9.6 & 12.0 \\
\hline & 20 & 3.5 & 171 & $\infty$ & 7.5 & 11.7 \\
\hline \multicolumn{7}{|c|}{$I_{p}=11 \mathrm{MA}$} \\
\hline \multirow[t]{2}{*}{$c_{r}=1.3$} & 20 & $\overline{2.2}$ & 36 & 8.9 & - & 8.5 \\
\hline & 40 & 3.3 & 80 & 10. & - & 8.3 \\
\hline \multirow[t]{2}{*}{$c_{\tau}=1.6$} & 20 & 3.8 & 106 & 266. (O $2 \mathrm{MW})$ & $=$ & 8.7 \\
\hline & 30 & 4.3 & 120 & $\infty$ & 7.8 & 8.5 \\
\hline \multirow[t]{2}{*}{$c_{r}=1.9$} & 10 & 4.3 & 135 & $\infty$ & 8.7 & 12.0 \\
\hline & 20 & 4.3 & 333 & $\infty$ & 7.5 & 9.3 \\
\hline \multicolumn{7}{|c|}{$I_{p}=13 \mathrm{MA}$} \\
\hline \multirow[t]{2}{*}{$c_{\tau}=1.0$} & 20 & 2.1 & 27 & 6.7 & - & 8.3 \\
\hline & 40 & 3.0 & 56 & 7.0 & . & 8.1 \\
\hline \multirow[t]{2}{*}{$c_{\tau}=1.3$} & 10 & 3.0 & 54 & 68. (@4 MW) & - & 10.9 \\
\hline & 30 & 4.6 & 151 & $\infty$ & 8.2 & 8.3 \\
\hline \multirow[t]{2}{*}{$c_{\tau}=1.6$} & 10 & 5.1 & 241 & $\infty$ & 8.9 & 12.0 \\
\hline & 20 & 5.1 & 492 & $\infty$ & 7.5 & 9.3 \\
\hline
\end{tabular}

Table I: Parameters for each of the 18 time-dependent simulations. 


\begin{tabular}{|c|c|c|c|c|c|}
\hline$I_{p}$ & $c_{-}$ & $P_{\text {aux }}$ & $W_{\text {OH }}$ & $W_{P_{\text {aus }}}$ & $t\left(0.95 W_{P_{\text {aus }}}\right)$ \\
\hline 9 & 1.6 & 20 & 5.5 & 30 & 8.3 \\
9 & 1.6 & 40 & 6.2 & 46 & 7.5 \\
11 & 1.3 & 20 & 6.4 & 33 & 8.5 \\
11 & 1.3 & 40 & 9.2 & 48 & 7.6 \\
13 & 1.0 & 20 & 8.6 & 28 & 7.7 \\
13 & 1.0 & 40 & 9.4 & 41 & 7.1 \\
\hline
\end{tabular}

Table II: Time required to heat plasma to $95 \%$ of $W_{P_{a \text { ax }}}$ evaluated using data from Table I and Eq. (22). 


\section{Figures}

FIG. 1. Time dependence of various CIT parameters taken from TSC simulation FilDi; units are given separately for each.

FIG. 2. Time dependence of the various terms in Eq. (2). The solid lines are the result of the 0-D calculation. The markers indicate corresponding values taken from TSC output. Note that several of these points have been deleted for clarity. The solid squares represent $P_{O H}$ from TSC; the diarronds are for $P_{\text {aur. }}$.

FIG. 3. Contours of constant alxiliary power in $M W$ in $\left\langle n_{t}\right\rangle$ and $\langle T\rangle$ space for the $11 \mathrm{MA}$ design with $c_{\tau}=1.6$. The density and $\beta$ limits are indicated.

FIG. 4. Time dependence of the various terms in Eq. (2) for the 11 MA configuration of CIT with $c_{e}=1.6$ and $\left\{n_{e}\right\}=3.8 \times 10^{20} \mathrm{~m}^{-3}$.

FIG. 5. Time dependence of the various terms in Eq. (2) for the $11 \mathrm{MA}$ configuration of CIT with $c_{-}=1.6$ and $\left\langle n_{e}\right\rangle=4.3 \times 10^{20} \mathrm{~m}^{-3}$.

FIG. 6. Time dependence of the various terms in Eq. (2) for the II MA configuration of CIT with $c_{t}=1.6$ (using the power- dependent form of $\left.\tau_{E}\right)$ and $\left\langle n_{e}\right\rangle=3.8 \times 10^{20} \mathrm{~m}^{-3}$.

FIG. 7. Time dependence of the various terms in Eq. (2) for the $11 \mathrm{MA}$ configuration of CIT with $c_{T}=1.6$ (using the power- dependent form of $\left.\tau_{E}\right)$ and $\left\langle n_{e}\right\rangle=4.3 \times 10^{20} \mathrm{~m}^{-3}$. 


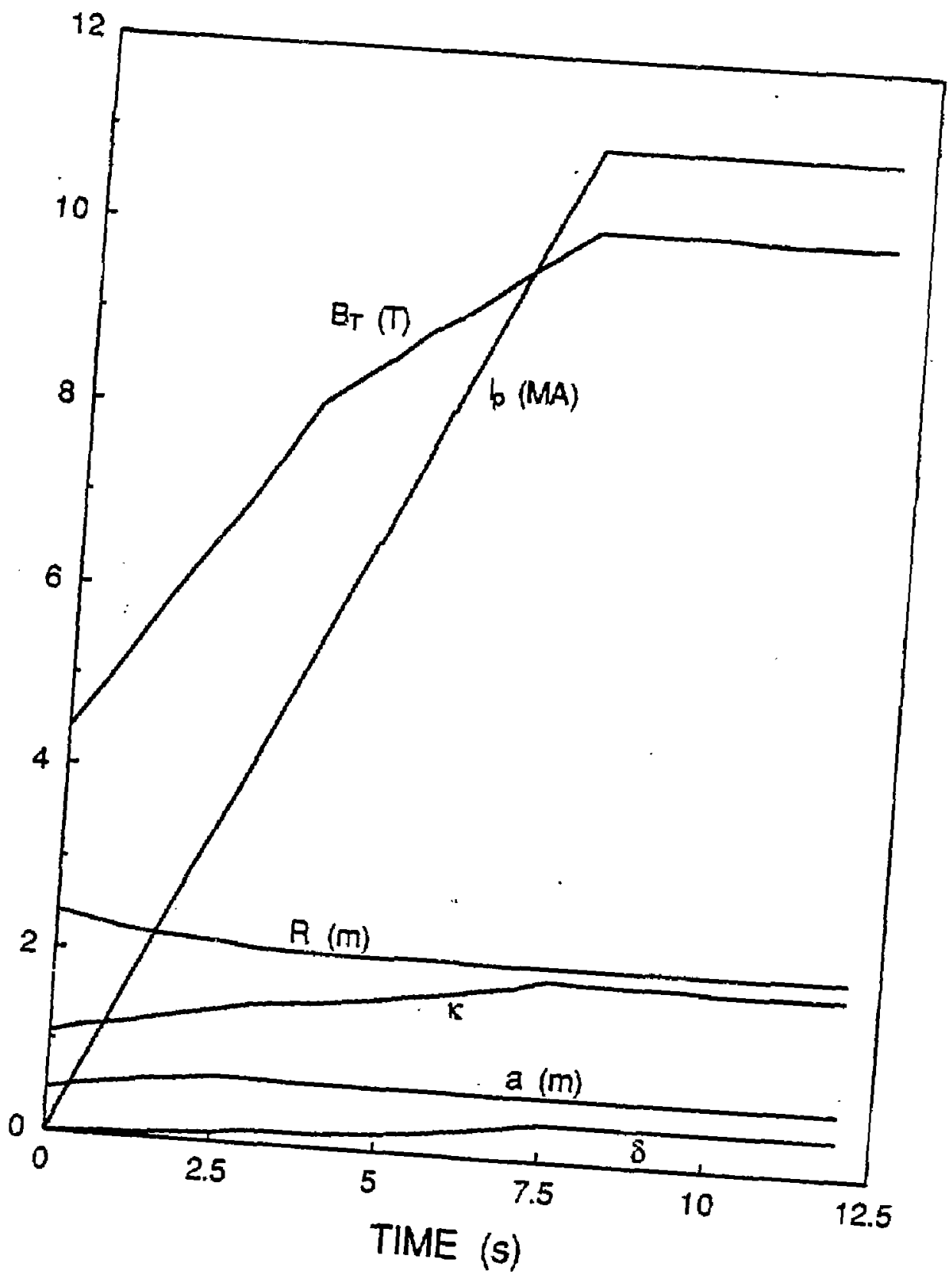

FIG. 1 


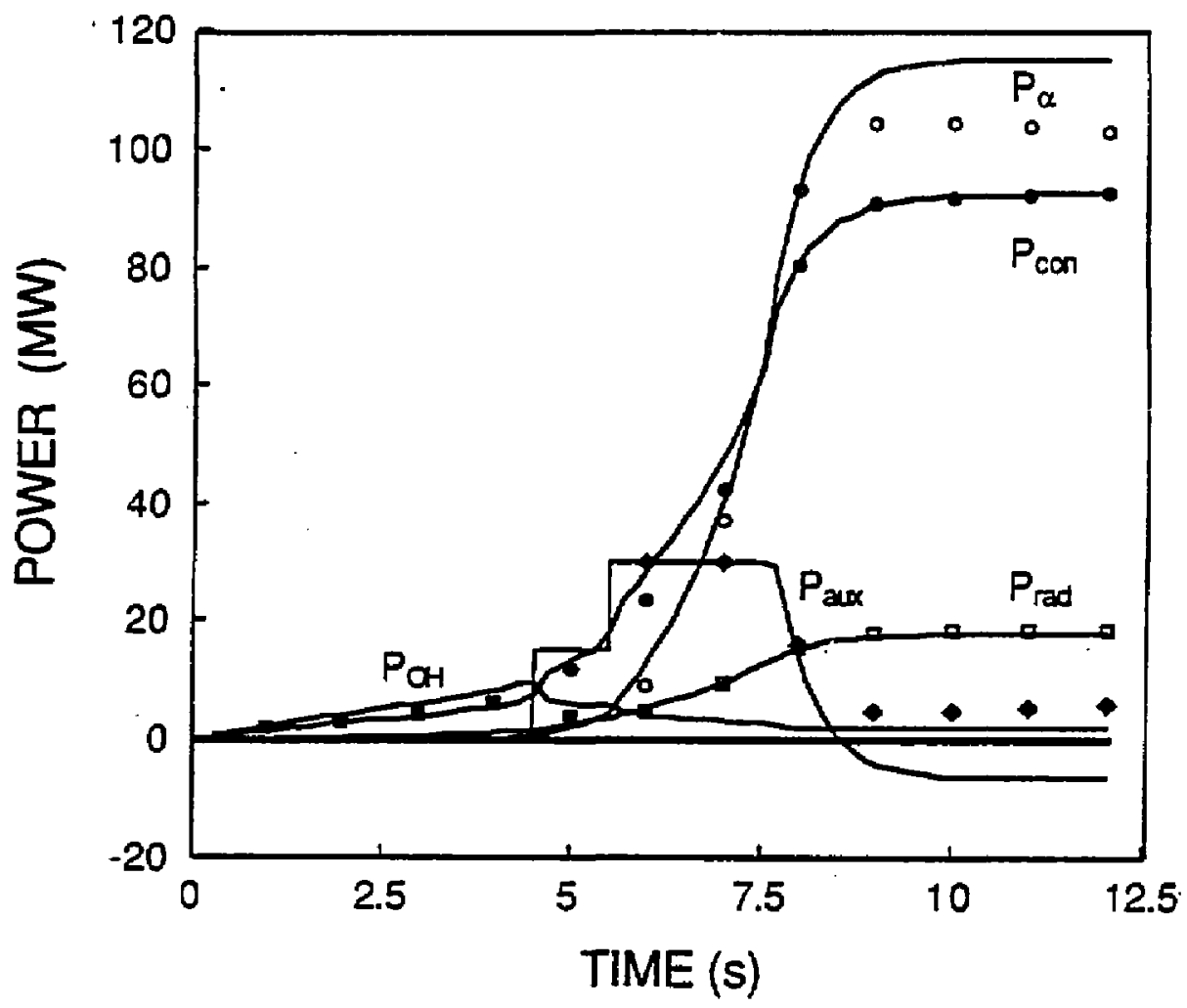

FIG. 2 


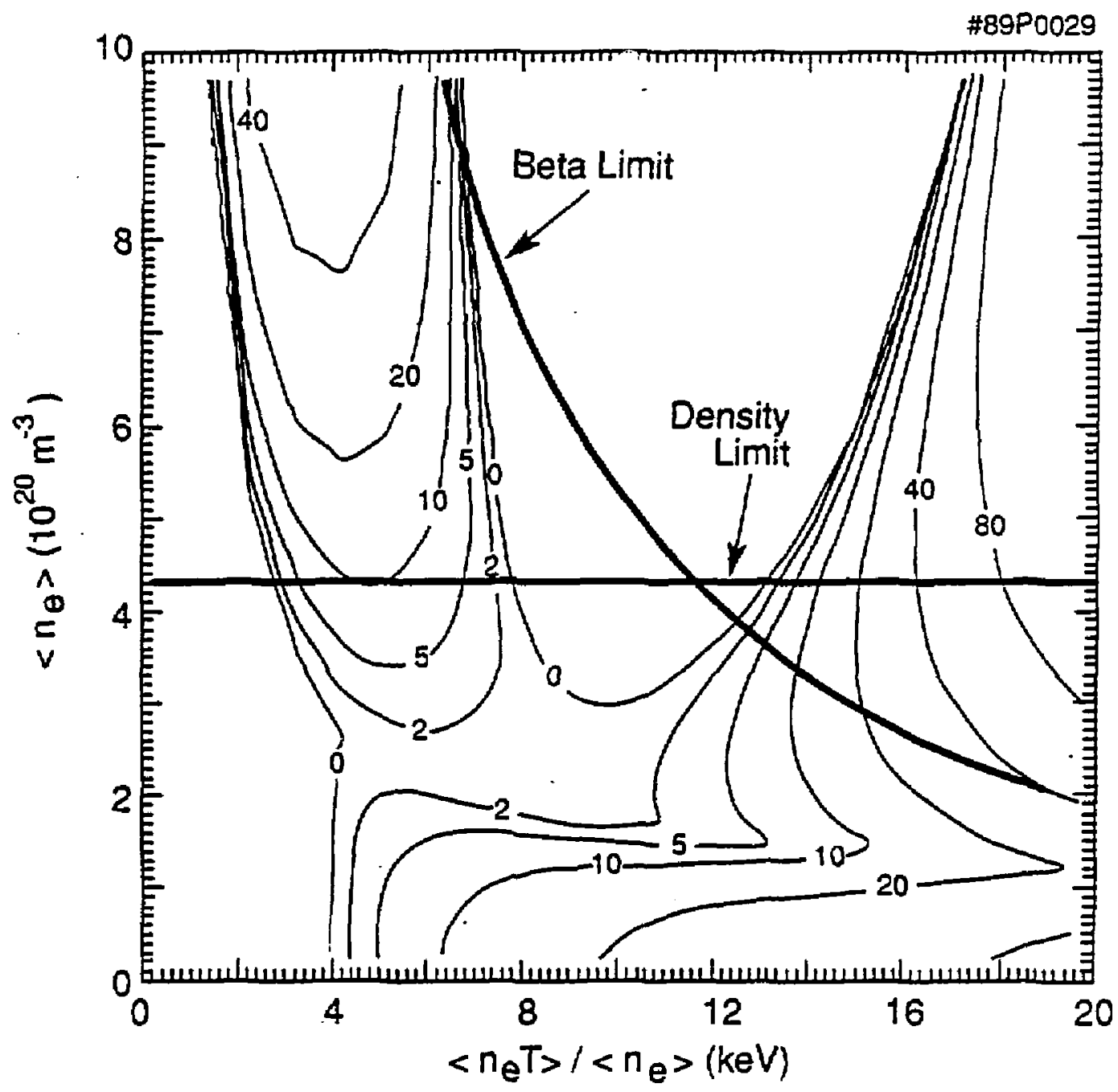

FIG. 3 


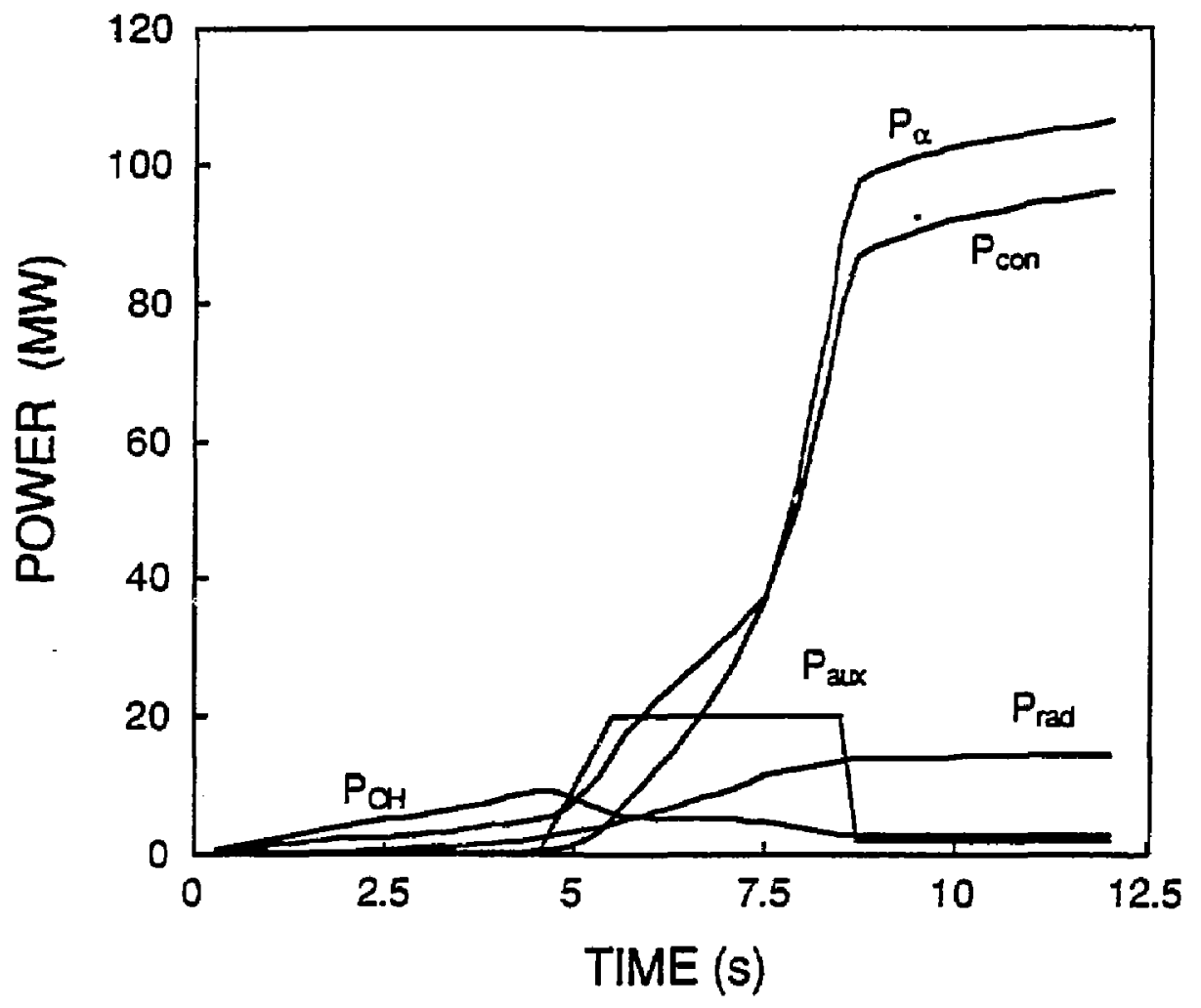

FIG. 4 


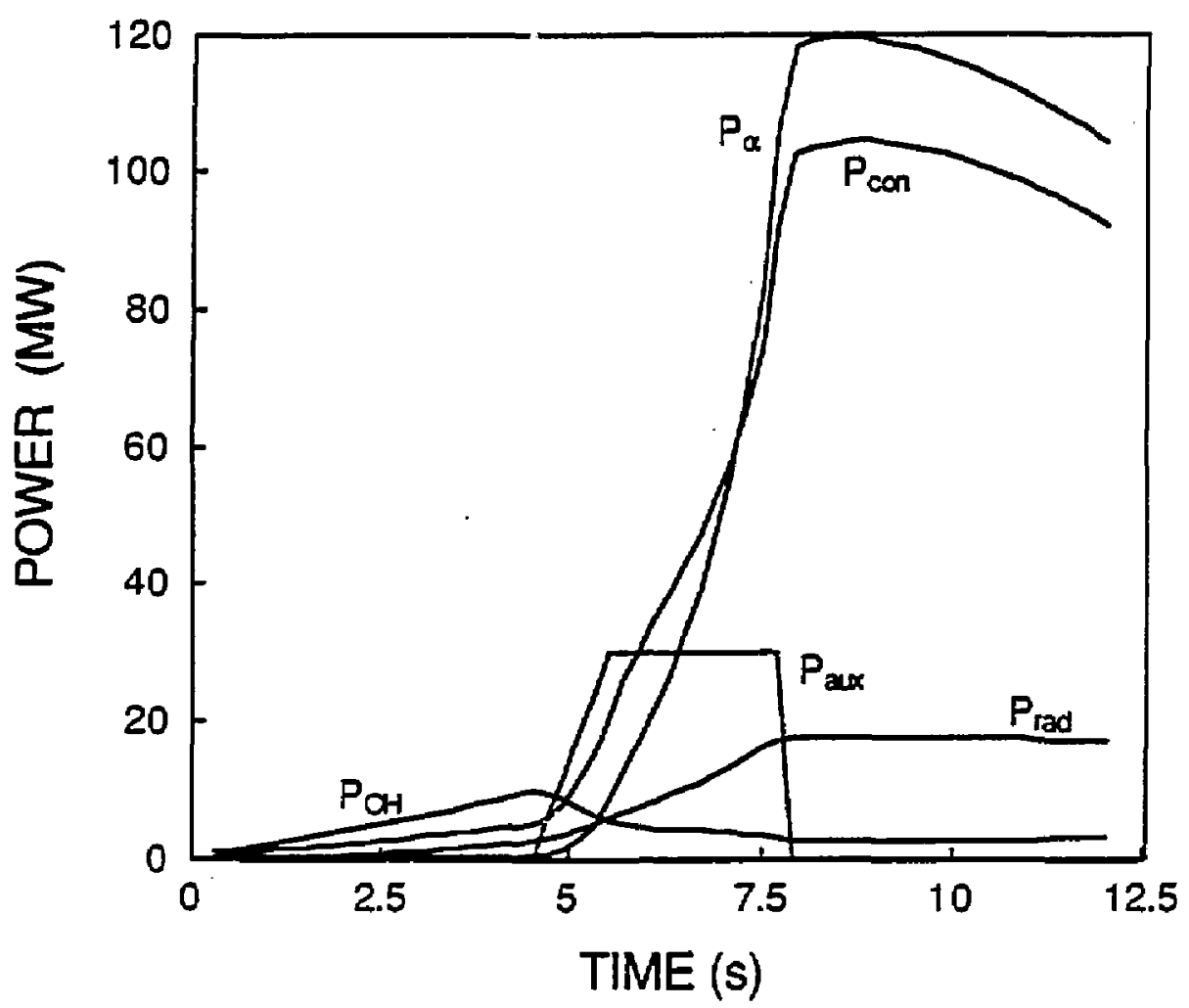

FIG. 5 


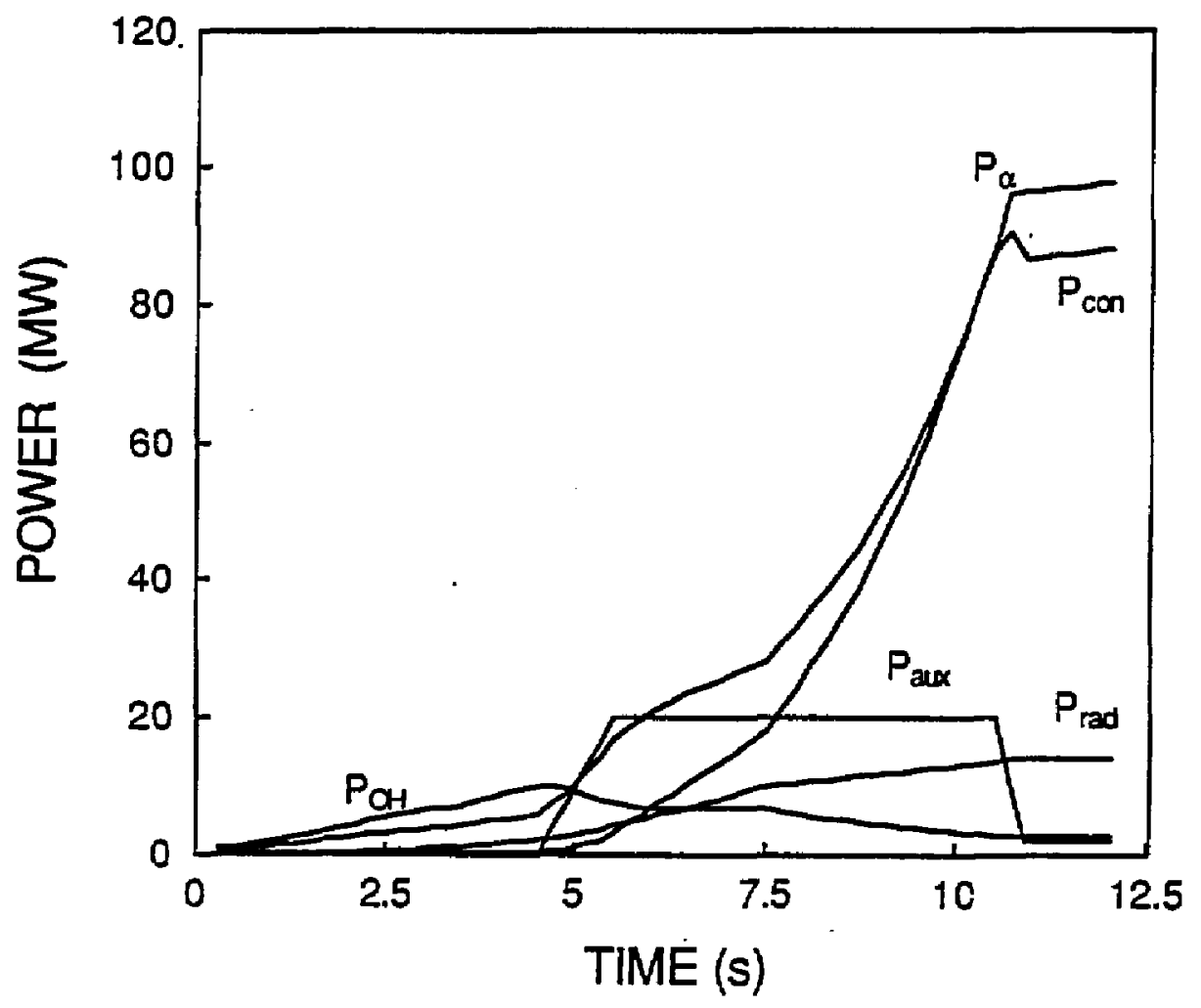

FIG. 6 


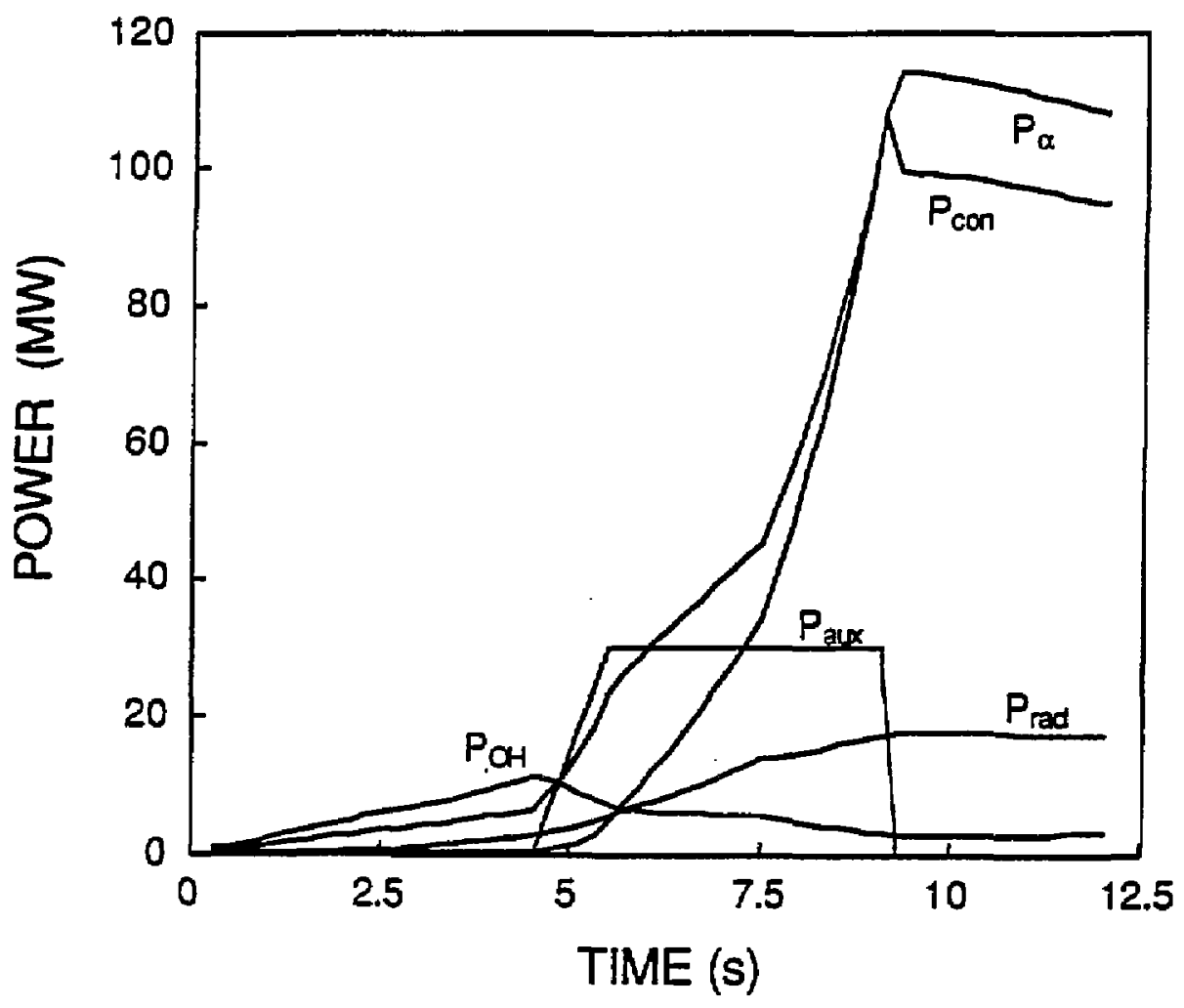

FIG. 7 
Dr. Frank J. Puoloni, Univ of wollangong, AUSTRALIA Prof. M.H. Brennan, Univ Sydney, Australia

Plasea Resaerch Lab., Australian Hat. Univ.., AUSTRALIA

Prof. I.R. Jones, Flindars UnlY., NUSTRALIA

Prof. F. Cap, Inst Theo Phys, MustRia

Prof. M, Helndler, Instut fur Theoretisehe Physik, AUSTRIA

M. Goossens, Astronow seh Instituut, EeLGIU,

Ecol a Royale Milltaire, Lob de Phys Piesens, Belgium

Comission-European, Og-XII Fusion Prog, BelgIUy

Prof. R. Bouciqu: Rijksuniversitelt Gent, BELGILM

Dr. P.H. Sakangka, Instituto Fisica, ERAzIL

Instituto os Pesquisas Espaciasi-INPE, ERAzIL

Decuments oftice, Atomic Energy of Canoda Limited, cuwba

Or. M.P. Bechynst, Ira Technologites, Ine., Cwana

Dr. H.M. Skersgord, University of Sasketcheman, CWwDA

Dr. H. Barnard, University of British Columbia, CMNDA

Prof. J. Teicheann, Univ. of Hontreel, CawDa

Prof. S,R. Sreanivasen, University of Calgary, Cuwan

Prof. Tudor W. Johnston, I Fas-Energie, Chwoh

Dr. Bolton, Contre cenadion de fusion aagnétique, Cawach

Or. C.R. Janes, Univ, of Albarta, CAkitan

Dr. Pater Lukac, Komenakeho Univ, CZECHOSLOVAKIA

The Librarien, Culten Leboratory, ENGLAND

The Librarien, Rutherford Appleton Leboratory, EkLLMD

Mrs. S.A. Hutchinson, JET LIbrery, ENELAD

C. Mouttet, Lab. o Physique des Mil ibux Ionisés. Frarce

J. Radet, CEN/CAOARACHE - Bat 506, France

us. C. Rinni. Librarian, Univ. of loannias, GREECE

Dr. Tom Muel, Acodery Bibliographic Ser., Hong kOnG

Preprint Library, Hungarian Acadary of Sciences, KUMGARY

Dr. B. Das Gupte, Sahe Inst of Nuci. Phys., INDIA

Dr. P. Kaw, Institute for Plasind Reseerch, INDIA

Dr. Philid fosenau, Israel Inst. of Tuch, ISRAEl

Librarian, InT'l Cer Thes Phys, ITALY

Prof. G. Rostagni, Istituto Gos Ionizzoti Del OR, ITALY

Miss Clelio De Palo, Assoc ElRATOM-ENEA, ITALY

Dr. G. Grosso, Istituto di fisica del Piasad, ITALY

Or. H. Taeato, Toshiba Ras \& Dav, JAPAN
Prof. 1. Kawakami, Atomic Energy Res. Institute, JAPAN

Prof. Kyoji Nishikama, Univ of Hiroshima, JAPAN

Director, Dept. Large Tokomak Res. JAEAI, JAPAN

Prof, Satoshi Itoh, Kyushu University, JAPAH

Resuarch Infa Conter, Nagoyo University, JAPAN

Prof. 5. Tanaka, Kyoto University, JAPAN

Library, Kyoto University, JAPAN

Prof. Nobuyuki Inoul, Univarsity of Tokyo, JAPAN

S. Mor $i$, JAERI, JAPAN

H. Jeong. Librerian, Karea Advaneed Energy Res Inst, KOREA

Prof. D.I. Choi, The Karea Adv. Inst of Sci \& Teeh, KOREA

Prof. 8.S. Lilay, Univarsity of Haikato, NEW ZEALANo

Institute of Plasma Physics, PEOPLE's REPUELIC OF CHIMA

Librarian, instltute of Phys., PEOPLE's RePliblic of CHINA

Library, Tsing Hue University, PEOPLE'S REPUBLIC OF CHINA

2. Li, Southwest Inst. Physics, PEOPLE's REPUalic of CHINA

Prof. J.A.C. Cabral, Inst Suderior Teenico, PORTUGal

Dr. Octavian Petrus, AL I CuzA University, ROMANIA

Dr. Jea de villiers, Fusion Studies, AEC, SO AFRICA

Prof. M.A. Hell lberg, University of Natal, SO AFRICA

C. I.E.M.A.T., Fusion Div. Library, SPAIN

Dr. Lennart Stentio, University of UMEA, SWEDEN

Liorary, Royal Institute of Tach, SWEDEN

Prot. Hans Wilhelmson, Chalmers Univ of Tech, SWEDEN

Contre Phys das Plasas, Ecole Polytech Fod, SHITZERLAND

Blbliothask, Fon-Inst Voor PIasma-Fysica, THE NETHERLANDS

Metin Ourgut, Middle East Technical University, TUAKEY

Or. O.D. Ryutov, Sibarian Acad Sci, USSR

Dr. G.A. El isoer, Kurchator Institute, USSR

Dr. V.A. Glukhikh, Inst Electrophysical Apparatus, USSR

Prof. 0.5. Padichenko, Inst. of Phys. A Tech. USSR

Dr. L.M. Kovrizhnykh, Institute of Gan. Physics, USSR

Nuctear Ros. Establishment, Julich Ltd., W. GEPAMNY

Blbliothek, Inst. Fur Plasmafarschung, H. GERMANY

Dr. K. Schindler, Ruhr-Universitat Bochua, H. GERAANY

ASDEX Reading Ren, c/o Wagner, IPPMax-Planck, W. GERMANY

Librorian. Mox-Planck Institut, W. GERMANY

Prot. R.K. Janev, Inst of Phys, yucoslavia 